\title{
Ag-grafted on ZnO nanorod arrays using UV-assisted irradiation for enhanced SERS behavior in CV detection
}

\author{
Ton Nu Quynh Trang ${ }^{1,2}$, Le To Cam Huong ${ }^{1,2}$, Thai Duong ${ }^{3}$, Vu Thi Hanh Thu ${ }^{1,2, *}$
}

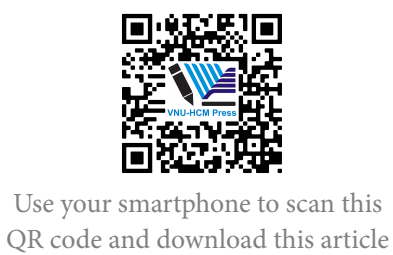

${ }^{1}$ Faculty of Physics and Physics Engineering, University of Science, Ho Chi Minh City 700000, Vietnam

${ }^{2}$ Vietnam National University, Ho Chi Minh City 700000, Vietnam

${ }^{3}$ Research Laboratories of Sai Gon Hi-Tech Park

\section{Correspondence}

Vu Thi Hanh Thu, Faculty of Physics and Physics Engineering, University of Science, Ho Chi Minh City 700000 Vietnam

Vietnam National University, Ho Chi Minh City 700000, Vietnam

Email: vththu@hcmus.edu.vn

History

- Received: 2021-02-19

- Accepted: 2021-05-09

- Published: 2021-05-13

DOI : 10.32508/stdj.v24i2.2519

\section{Check for updates}

\section{Copyright}

(c) VNU-HCM Press. This is an openaccess article distributed under the terms of the Creative Commons Attribution 4.0 International license.

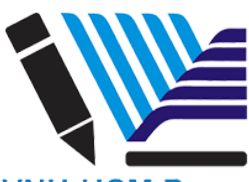

VNU-HCM Press

\begin{abstract}
Introduction: Semiconductor-based surface-enhanced Raman scattering (SERS) substrates with high stability and reproducibility have become one of the essential analytical tools in the analysis of chemical and biological at trace levels. Herein, a growth of the hexagonal-wrapped ZnO nanorod arrays decorating with Ag nanoparticles (AgNPs) at different concentrations of Ag was proposed. Methods: The crystallinity, morphology, chemical composition, and optical properties of the prepared samples were investigated by X-ray powder diffraction (XRD) and field emission scanning electron microscopy (FE-SEM), energy-dispersive X-ray spectroscopy (EDX), Raman system, respectively. Results: The results revealed that the SERS performance of ZnO NRs incorporating with AgNPs exhibited higher detection of crystal violet (CV) probe molecules at a low concentration of $10^{-8} \mathrm{M}$ than that of the pristine $\mathrm{ZnO}$ NRs. This effect originates from the localized surface plasmonic resonance of AgNPs that could cause a strong electromagnetic field and synergistic effects of Ag, ZnO, and CV molecules in ZnONRs@Ag/CV SERS system. Conclusion: These outcomes reveal that AgNPs play a crucial role in enhanced SERS performance for chemical and biological detection of ZnO substrate.
\end{abstract}

Key words: AgNPs, ZnO nanorod arrays, surface-enhanced Raman scattering (SERS), electromagnetic mechanism, chemical mechanism

\section{INTRODUCTION}

In recent decades, surface-enhanced Raman scattering (SERS) is a powerful spectroscopic analytical tool that enables the identification and characterization at trace detection of chemical and biological molecules due to its attractive properties such as extremely rapid, ultra-sensitive and fingerprint diagnostics, non-destructive data acquisition. The basic concept of SERS is that the amplification of the signals of the analyte is created by the interaction between the substrate, target molecules, and lights on the surface. The SERS effect is based on two main mechanisms: chemical mechanism $(\mathrm{CM})^{1}$ and electromagnetic mechanism (EM) enhancements ${ }^{2}$. The EM enhancement is obtained by the electric field induced the localized surface plasmonic resonance (LSPR) related to noble metal nanostructures with a typical enhancement factor of $10^{4}-10^{7}$ folds, while the CM enhancement is directly correlated with the charge transfer process between adsorbed molecules and the substrate materials such as semiconductors with enhancement factors in the range 10-100 times ${ }^{3,4}$. Most of these studies reported that plasmonic metal nanoparticles (NPs) were employed for SERS; it supplies highly sensitive detections based on the enhancement of the EM. The strong near-field electro- magnetic amplifications depend on the shapes, sizes, composition, number of NPs, and position of Raman reporters at the surface ${ }^{5-7}$. Despite recent progress in SERS applications of noble metals, their practical application has been hindered by the uniformity, reproducibility, and instability with easy aggregation and oxidation and the high cost ${ }^{8,9}$. To overcome these drawbacks, most efforts have gone into developing flexible and uniform SERS substrates ${ }^{10-12}$, a promising alternative approach for designing hybrid ternary nanocomposite by combining noble metals and semiconductor materials has been proposed. SERS performance based on semiconductors is an attractive characteristic such as low cost, resource abundance, high chemical stability, biocompatible and piezoelectric properties. However, these strategies face a significant challenge because the SERS sensitivity of these semiconductor substrates is relatively low, becoming a bottleneck in the semiconductor SERS development. Therefore, it is vital to develop SERS semiconductor substrates with high sensitivity, low cost, uniformity, and reproducibility. Compared with other metal oxide materials, Zinc oxide ( $\mathrm{ZnO}$ ) has been intensively carried out for SERS because of its enrichment, economical fabrication, nontoxicity, and photochemical stability ${ }^{13,14}$. Plasmonic nanoparticles are deposited 
on the semiconductor material's surface and have been an efficient pathway for enhancing the SERS activity ${ }^{15,16}$. When the $\mathrm{ZnO}$ is connected with plasmonic metals, it provides the LSPR of metals and exhibits the charge-transfer process in the SERS system between semiconductors, noble metal nanomaterials, and target molecules. Therefore, both EM and CM enhancements may be observed on these hybrid SERS substrates, leading to a significant SERS performance. Recently, $\mathrm{ZnO}$ nanorods (NRs) array increasing the surface area to decorate plasmonic structures for enhancing Raman scattering have been easily grown via hydrothermal procedure on any substrates ${ }^{17}$. Moreover, the nanostructures with highly ordered arrangement can improve the light absorption capability that could further promote the robust enhancement effects of SERS ${ }^{18}$.

In view of the above considerations, a $1 \mathrm{D} \mathrm{ZnO}$ nanorods decorated with Ag NPs were fabricated using a facile and low-cost approach for SERS performances in this work. The growth of $\mathrm{ZnO} N R$ arrays on $\mathrm{ZnO}$ seed layer using hydrothermal method and Ag NPs deposition on the surface of $\mathrm{ZnO}$ NRs by photoreduction method were proposed by UVassisted irradiation. The synthesized Ag/ZnO NRS substrate shows good SERS activity at a low concentration of $\mathrm{CV}$ molecules compared to the pristine $\mathrm{ZnO}$. A mechanism of SERS activity of Ag/ZnO NRs active-platform is proposed.

\section{MATERIALS - METHODS}

\section{Materials}

Zinc nitrate hexahydrate $\left(\mathrm{Zn}\left(\mathrm{NO}_{3}\right)_{2} \cdot 6 \mathrm{H}_{2} \mathrm{O}\right.$, 99\%, Merck, United States), crystal violet (CV, $\mathrm{C}_{25} \mathrm{H}_{30} \mathrm{ClN}_{3}, 99 \%$, Merck, United States), hexamethylenetetramine (HMTA, $\mathrm{C}_{6} \mathrm{H}_{12} \mathrm{~N}_{4}, 99 \%$, Merck, United States), hydrochloric acid ( $\mathrm{HCl}, 38 \%$, SigmaAldrich, United States), ethanol solution $\left(\mathrm{C}_{2} \mathrm{H}_{5} \mathrm{OH}\right.$, $<$ 99.5\%, Merck, United States), ZnO target (99\%, Singapore Advantech, Singapore) silver nitrate $\left(\mathrm{AgNO}_{3}, 99 \%\right.$, Merck, United States). De-ionised (DI) water was utilized in all experiments. Glass wafers were employed to fabricate the SERS-active platform.

\section{Synthesis of ZnO nanorod arrays (NRs)@Ag}

A $\mathrm{ZnO}$ growth layer was fabricated on the glass slides using RF magnetron sputtering. Before the deposition, $1 \mathrm{~cm} \mathrm{x} 1 \mathrm{~cm}$ glass slides were washed by ultrasonication in an aqueous solution of $\mathrm{C}_{2} \mathrm{H}_{5} \mathrm{OH}$ and $\mathrm{HCl}$ and dried with nitrogen flux. Then, the $\mathrm{ZnO}$ growth layer was deposited at a working pressure of $3 \times 10^{-3}$
Torr at $70 \mathrm{~W}$ in an Ar flow for deposition times 70 min. Afterward, ZnO NRs were fabricated by the hydrothermal method. A typical process, was placed the growth solution containing $25 \mathrm{mM} \mathrm{Zn}\left(\mathrm{NO}_{3}\right)_{2} \cdot 6 \mathrm{H}_{2} \mathrm{O}$ and $25 \mathrm{mM} \mathrm{C}_{6} \mathrm{H}_{12} \mathrm{~N}_{4}$ with an equivalent concentration of $25 \mathrm{mM}$ dissolved in $200 \mathrm{~mL}$ of DI water was stirred for $60 \mathrm{~min}$ at room temperature. The achieved mixture was transferred into an autoclave with Teflonliner. The $\mathrm{ZnO}$ growth layer was vertically immersed into the solution. The autoclave was placed in an oven at $90^{\circ} \mathrm{C}$ for 7 hours. After the growth duration, the solution was cooled at room temperature. The resulted sample was carefully rinsed with double-distilled water to remove contaminations on the surface and dried under an $\mathrm{N}_{2}$ environment. After that, the decoration of AgNPs on the ZnO NRs platform was obtained by UV-assisted illumination. The aqueous solution of $\mathrm{AgNO}_{3}$ was prepared by adding $\mathrm{AgNO}_{3}$ at different concentrations of $\mathrm{Ag}$ (amounts of $\mathrm{AgNO}_{3}: 0.5 \mathrm{wt} . \%$, $1.0 \mathrm{wt} . \%$, and $2.0 \mathrm{wt} . \%$.) into the $5 \mathrm{~mL}$ double-distilled water and stirred in $10 \mathrm{~min}$. Next, the ZnO NFs substrate was immersed in the above aqueous solution using UV-assisted irradiation. After being decorated with AgNPs, the obtained samples were thoroughly washed with double-distilled water and then dried in $\mathrm{N}_{2}$ environment. The ZnONFs@Ag substrate was engineered on the $\mathrm{ZnO}$ growth layer throughout procedures as presented in Figure 1.

\section{Material characterization}

The morphologies of the ZnONFs@Ag substrate were characterized using a field emission scanning electron microscope (FESEM, Hitachi) equipped with energy dispersive spectroscopy (EDS) spectra. In addition, the crystalline structure of specimens was investigated by an X-ray diffractometer (XRD, D8, ADVANCE, BURKER). SERS spectra were carried out using a green laser of $532 \mathrm{~nm}$ wavelength with a laser power of $1 \mathrm{~mW}$ and an integration time of $10 \mathrm{~s}$ (Horiba XploRA PLUS). For SERS measurement, CV was used as the target molecules with various concentrations (from $10^{-3}$ to $10^{-8} \mathrm{M}$ ), dropped onto the SERS platform, and then dried under room temperature before the evaluation.

\section{RESULTS}

The crystalline structure of the as-prepared samples was characterized by X-ray diffraction (XRD) spectroscopy, as illustrated in Figure 2. The diffraction peak at $2 \theta=35^{\circ}$ can be assigned to the (002) crystal plane of hexagonal wurtzite $\mathrm{ZnO}$ (JCPDS card No. 36-1451). The strong and dominant peak in the XRD analysis is preferential growth along the c-axis of the 


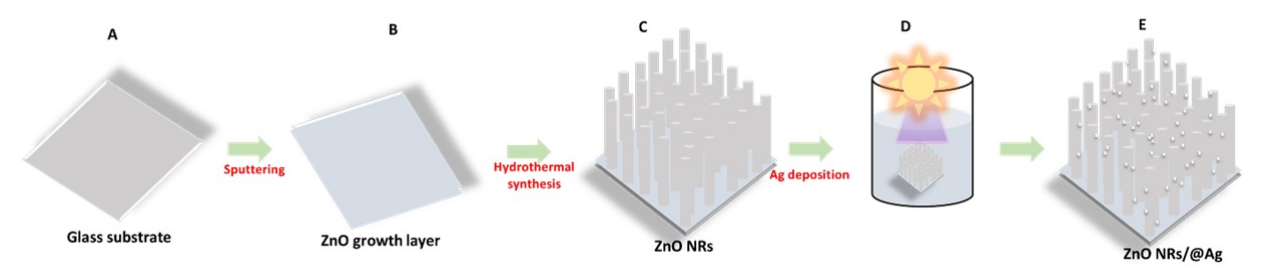

Figure 1: Schematic for the fabrication of the ZnONRs@Ag platform. (A) Preparation of the glass substrate. (B) Covering $\mathrm{ZnO}$ growth layer through magnetron sputtering. (C) The obtained ZnO NRs sample after synthesized by hydrothermal method. (D) the decoration of Ag NPs onto the surface of ZnO NRs via photoreduction approach. (E) Ag assembled onto the ZnO NRs platform.

ZnO nanorod arrays. No other peaks were recorded. The other diffraction peak (labeled with ${ }^{\star}$ ) at $2 \mathrm{q}$ value of $38^{\circ}$ maybe indexed as the diffraction peak of the face-centered cubic (fcc) Ag peaks (JCPDS card No. 04-0783) related to the (111) plane. This implies that the AgNPs are well-formed on the surface of $\mathrm{ZnO} N R s$ with no contamination.

The morphological characteristic of the assynthesized ZnO NRs and ZnONRs@Ag was investigated via SEM images (Figure 3). As shown in Figure 3a,b, the $\mathrm{ZnO}$ nanorod arrays are vertically grown with an average diameter of $\sim 50 \mathrm{~nm}$. The growth of $\mathrm{ZnO} \mathrm{NRs}$ oriented onto the $\mathrm{ZnO}$ seed layer can supply an enhanced SERS performance based on the photon scattering and charge transfer process of the $\mathrm{ZnO}$ NRs. Figure $3 \mathrm{c}$ shows the morphology of AgNPs anchored on the ZnO NRs surface. As shown in Figure 3c, these $\mathrm{Ag}$ nanoparticles are anchored on the surface of $\mathrm{ZnO}$ NRs with a diameter of approximately $\sim 20 \mathrm{~nm}$, leading to an enhanced SERS behavior due to the strong electric field effects related to the EM. Next, to further determine the Ag NPs' composition onto the $\mathrm{ZnO}$ nanorods, EDS-mapping analysis of the sample was carried out as depicted in Figure. Figure 3d-g shows the presence of $\mathrm{O}$ (red), $\mathrm{Ti}$ (green), and Ag (blue). Additionally, the EDS spectrum (Figure $3 \mathrm{~h}$ ) proves proof associated with the corresponding elemental mapping for $\mathrm{Zn}$, O, and Ag, suggesting that AgNPs decorated successfully on the surface of the $\mathrm{ZnO}$ NRs.

To evaluate the vibration modes of the as-obtained samples, Raman spectra of the pristine ZnO NRs and $\mathrm{Ag}$ decorated $\mathrm{ZnO}$ NRs as illustrated in Figure 4. It is noted that the vibration mode attributed to the $\mathrm{ZnO}$ nanorods is at $438 \mathrm{~cm}^{-1}$, which corresponds to the E2 optical phonon band ${ }^{19}$. Moreover, it is seen that the intensity of the pristine $\mathrm{ZnO}$ NRs is very weak, when the presence of Ag induces a high intensity of $\mathrm{ZnO} \mathrm{Ra}-$ man signal, implying an enhanced SERS performance in $\mathrm{ZnONRs/Ag} \mathrm{platform,} \mathrm{and} \mathrm{the} \mathrm{main} \mathrm{peak} \mathrm{of} \mathrm{ZnO}$ NRs has maintained. This outcome suggests that the deposition of AgNPs onto the surface of $\mathrm{ZnO}$ NRs is not significant change any phase structure and vibrational modes.

The SERS sensing of the pristine $\mathrm{ZnO}$ NRs and the hybrid nanostructures of Ag-decorated ZnO NRS was evaluated using Crystal Violet (CV) as a target molecule under the excitation wavelength of 532 nm. The SERS spectra of CV are presented in Figure 5a. A significant difference between the intensity of signals assessed on the glass, pristine $\mathrm{ZnO}$ NRs, and Ag-decorated ZnO NRs platform. The Ag-decorated $\mathrm{ZnO}$ NRs platform exhibits the highest SERS signals among these substrates due to the synergistic effects between $\mathrm{Ag}, \mathrm{ZnO}$, and R6G. No Raman signals of the $\mathrm{CV}$ molecules are recorded using the glass and the $\mathrm{ZnO}$ NRs substrate due to the large forbidden of $\mathrm{ZnO}$ NRs. All the typical characteristic peaks of the $\mathrm{CV}$ molecules centered at $(526,561$ $\left.\mathrm{cm}^{-1}\right), 729 \mathrm{~cm}^{-1}, 804 \mathrm{~cm}^{-1}, 916 \mathrm{~cm}^{-1}, 1174 \mathrm{~cm}^{-1}$, $1383 \mathrm{~cm}^{-1}, 1537 \mathrm{~cm}^{-1}$, and $1620 \mathrm{~cm}^{-1}$ could be assigned to the $\mathrm{C}-\mathrm{N}-\mathrm{C}$ antisymmetric bending, $\mathrm{C}-\mathrm{N}$ $\mathrm{C}$ symmetric stretching, Phenyl-H out-of-plane antisymmetric bending, Phenyl ring breathing mode, $\mathrm{C}-\mathrm{H}$ in-plane antisymmetric stretching, Phenyl-Cphenyl antisymmetric stretching, Phenyl-N antisymmetric stretching, and C-phenyl in-plane antisymmetric stretching, respectively ${ }^{20,21}$. The results indicate that $\mathrm{ZnONRs/Ag}$ is responsible for amplification of SERS signals based on i) plasmonic hot spots that can efficiently focus electromagnetic fields at/near the metal-semiconductor interface; ii) the charge transfer process between Fermi levels of the ZnO NRs, $\mathrm{Ag}$, and CV molecules; and iii) the chemical interactions between semiconductor and molecules related to matching of the energy structure band in the system. 


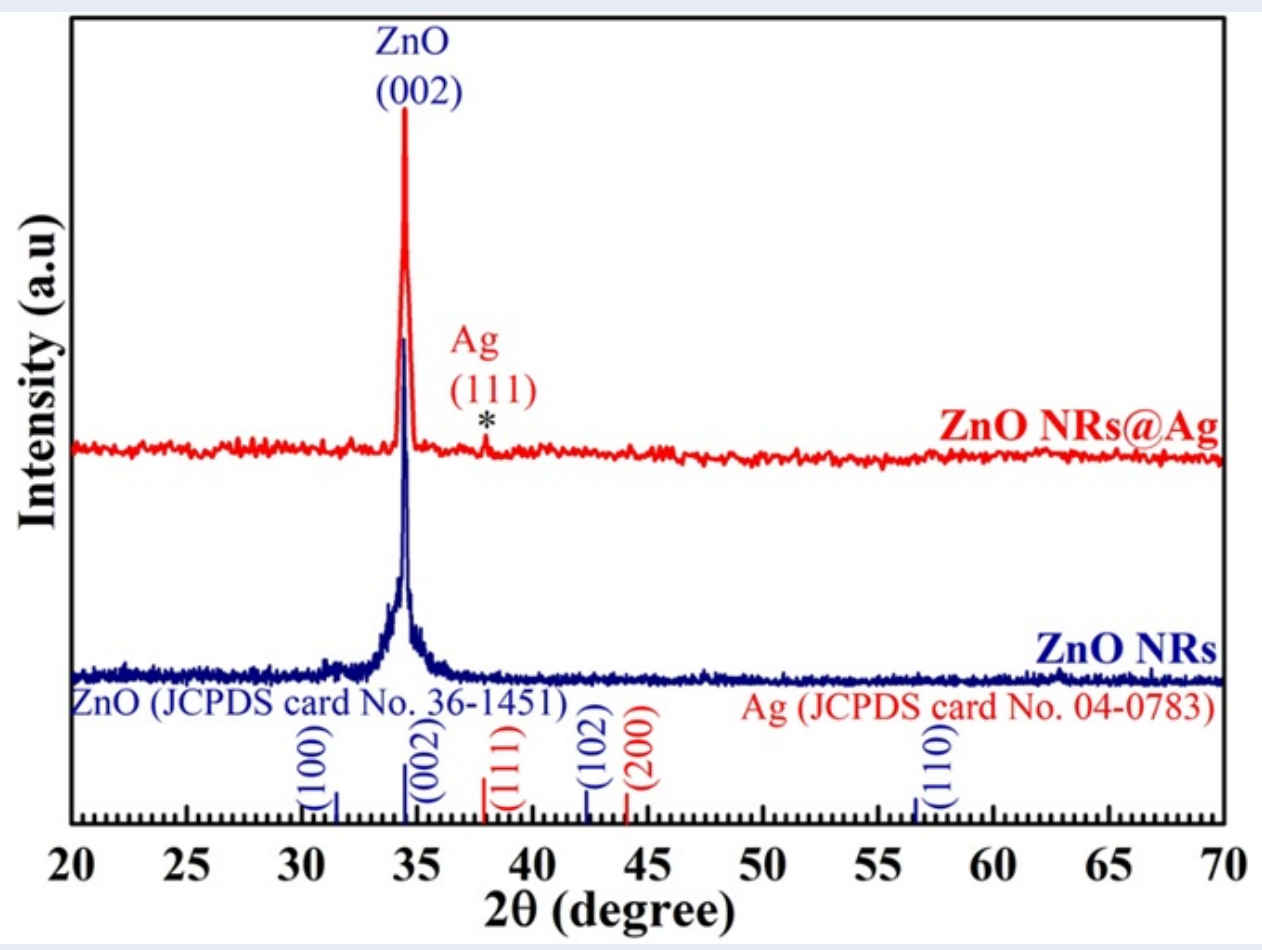

Figure 2: The crystallinity through XRD patterns of the pristine ZnO and ZnONRs@Ag substrate.

To further understand the role of Ag NP concentration on the SERS performance, the sensitivity of the ZnONRs/Ag samples with different Ag loadings was exposed in $10^{-6} \mathrm{M} \mathrm{CV}$ solution. As shown in Figure $5 \mathrm{~b}$, all the typically characteristic peaks of $\mathrm{CV}$ molecules could be detected at concentrations of $10^{-7}$ $\mathrm{M}$, suggesting a good SERS signal in the ZnONRs/Ag platform. The Raman intensities of the obtained platforms were gradually increased with increasing $\mathrm{Ag}$ content. The SERS signal of the $1.0 \mathrm{wt}$.\% Ag has the highest intensity. The lowest SERS signals are obtained after using of $\mathrm{ZnONRs/Ag}$ at $2.0 \mathrm{wt} . \%$, indicating that an excess amount of Ag deposition is not favorable for SERS activity. At an Ag concentration of $0.5 \%$, the density of the hot spots is low, leading to a weak Raman signal of the substrate. When the Ag concentration is $1.0 \mathrm{wt} \%$, many AgNPs and high-density nanogaps between adjacent AgNPs decorated homogenously onto the $\mathrm{ZnO}$ surface can cause a strong local electric field enhancement. Therefore, the SERS signal is significantly boosted. However, the SERS signals decline sharply when the Ag concentration is $2.0 \mathrm{wt}$.\%. This would be assigned to the decrease in the electromagnetic field distribution due to the formation of larger Ag NPs on the ZnO NRs surface caused by the merging of the adjacent AgNPs.
These results also suggested that adjusting Ag concentration in ZnONRs/Ag system are favorable for improving SERS activity owing to a change in the local electromagnetic field. In this paper, the $\mathrm{ZnONRs/Ag}$ at $1.0 \mathrm{wt} . \%$ is chosen as the optimal SERS substrate to assess the SERS activity further.

Then, the sensitivity of $\mathrm{ZnONRs/Ag}$ at $1.0 \mathrm{wt} . \%$ was carried out Raman enhancement ability at different CV molecule concentrations as depicted in Figure 6. The R6G characteristic peaks are distinguished even at a low concentration of $10^{-8} \mathrm{M}$ and gradually decreased with the decreasing R6G concentration, indicating good SERS sensitivity of the ZnONRs/Ag platform.

\section{DISCUSSION}

Herein, to further understand the SERS performance, energy structure band, and a charge-transfer process in ZnONRs/Ag system are critical to explaining SERS enhancement (Figure 7). The position of the energy levels of the highest occupied molecular orbital/lowest unoccupied molecular orbital (HOMO/LUMO) of the Raman probe, the CB and VB level of $\mathrm{ZnO} N R s$, and the Fermi energy level $\left(\mathrm{F}_{F}\right)$ of the Ag NPs plays a significant role in the charge-transfer process between SERS platform and 


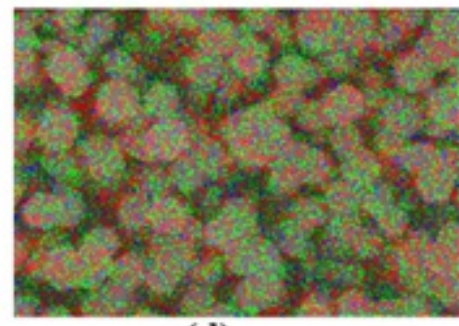

(d)

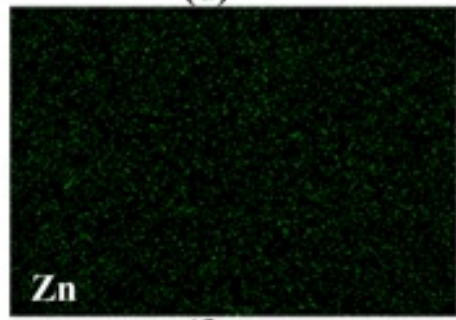

(f)

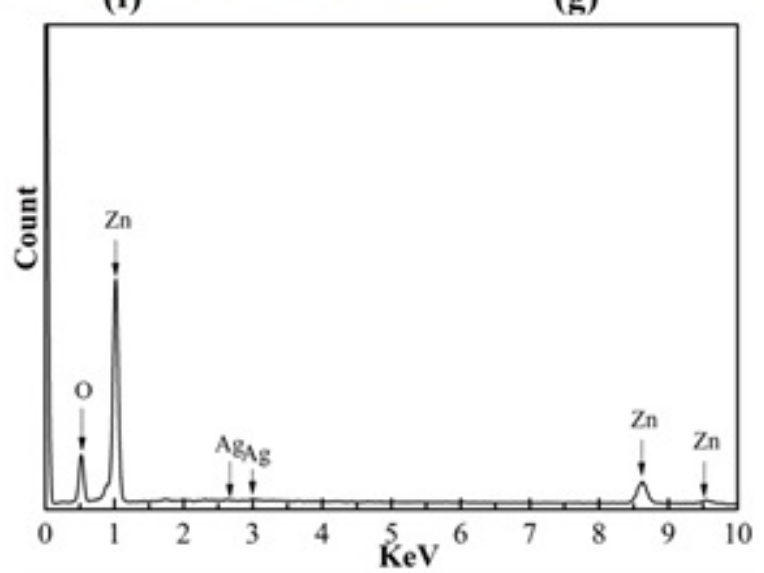

(h)

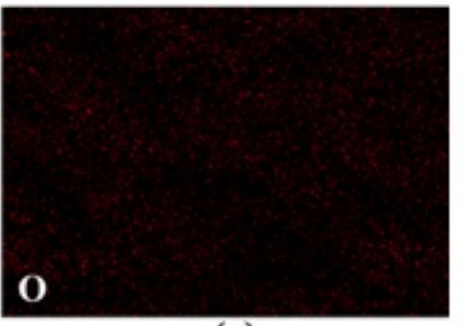

(e)

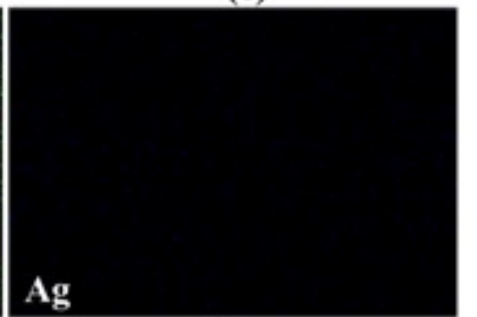

(g)

Figure 3: Morphological characteristics and elemental composition of the obtained ZnONRs@Ag substrate. (a,b) SEM images of ZnO NRs, (c) SEM image of ZnONRs@Ag, (d) SEM-elemental mapping of ZnONRs@Ag, (e-g) EDS-mapping of O (red), Zn (green), and Ag (blue), respectively, (h) EDS energy spectrum of the ZnONRs@Ag.

molecules. As shown in Figure 7, when CV molecules are employed as target molecules, the HOMO and LUMO level is at -6.0 and $-4.1 \mathrm{eV}$, respectively ${ }^{22}$. The position of $\mathrm{VB}$ and $\mathrm{CB}$ of $\mathrm{ZnO}$ is centered at -7.7 $\mathrm{eV}$ and $-4.5 \mathrm{eV}$, respectively. $\mathrm{F}_{F}$ of AgNPs is -4.84 $\mathrm{eV}$. Under green laser illumination of $532 \mathrm{~nm}$, hot charge carriers of the Ag are produced and jumped to the LUMO level of the CV molecules. Therefore, the chare-transfer process from $\mathrm{F}_{F}$ to the LUMO level of the $\mathrm{CV}$ occurred at $0.74 \mathrm{eV}$. Furthermore, the hot charge carriers are transported from the $\mathrm{F}_{F}$ to the $\mathrm{CB}$ at $0.34 \mathrm{eV}$ and shifted to the LUMO level at $0.4 \mathrm{eV}$ that could promote the charge-transfer activity and further enhance SERS behavior. It can be concluded that the design of AgNPs, $\mathrm{ZnO}$ NRs, and $\mathrm{CV}$ probe molecules in the platform and the induced "hot charge carriers" of AgNPs based on LSPR are accountable for the enhanced SERS performance.

\section{CONCLUSION}

In summary, a pathway for the preparation of the SERS platform as depicted in this work. The asfabricated $\mathrm{ZnONRs}$ with growth on the $\mathrm{ZnO}$ seed layer and decorated $\mathrm{Ag}$ on the surface of $\mathrm{ZnO} \mathrm{NRs}$ without contaminations have been easily prepared by facile and cost-effective approaches, in which the $\mathrm{ZnO}$ NRs were prepared through the hydrothermal method and then loaded AgNPs by photoreduction approach. A high detection of SERS substrate composing of a hexagonal structure of $\mathrm{ZnO}$ NRs deco- 


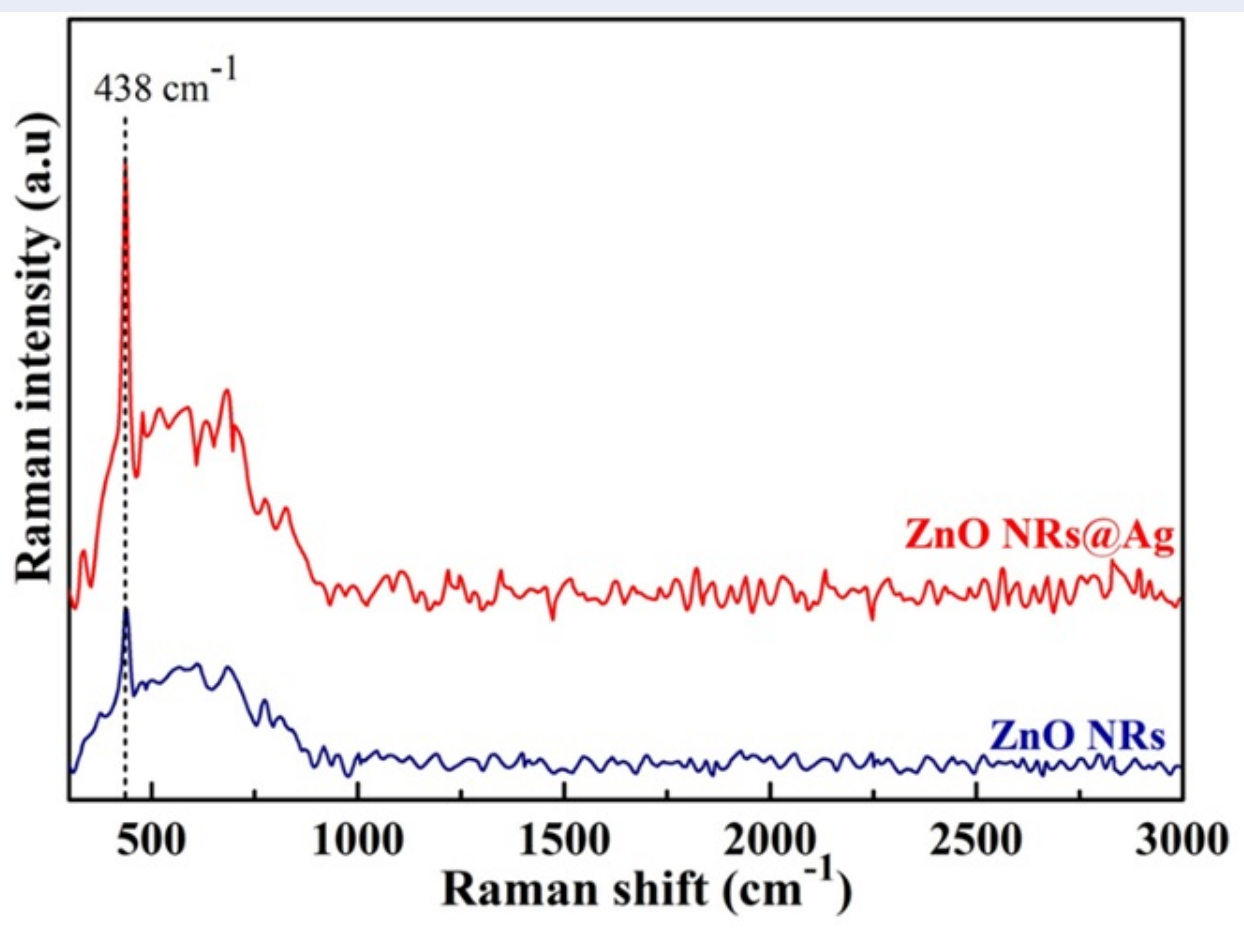

Figure 4: The vibrational modes through Raman spectra of the pristine ZnONRs and ZnONRs@Ag substrate.
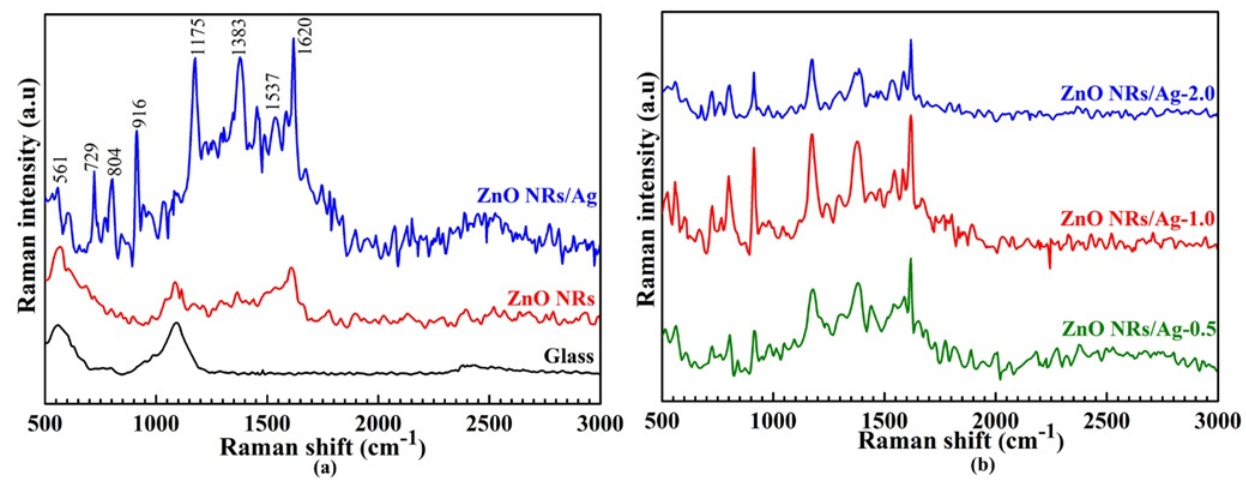

Figure 5: SERS signal spectra of CV molecules (a) on the glass, the pristine ZnO NRs, and the ZnONRs@Ag platforms, respectively, (b) on the ZnONRs@Ag at different concentrations of Ag from 0.5 to $2.0 \mathrm{wt} . \%$.

rated with AgNPs was fabricated through two steps: $\mathrm{ZnO}$ nanorod array was grown via hydrothermal procedures. Then, AgNPs were grafted onto the surface of $\mathrm{ZnO}$ NRs using the photoreduction method. Compared to pristine $\mathrm{ZnO} \mathrm{NRs}$, the highly sensitive ZnONRs@Ag active-platform in CV detection at a low concentration from $10^{-6} \mathrm{M}$ to $10^{-8} \mathrm{M}$ was exhibited. Especially, the optimal ZnONRs@Ag substrate at Ag concentration of 1 wt.\% could be wellidentified characteristic peaks of CV target molecules even ultra-low concentration of $10^{-8} \mathrm{M}$. This performance could be attributed to the i) plasmonic hot spots of AgNPs related to the EM; ii) the charge transfer between $\mathrm{ZnO} \mathrm{NRs}, \mathrm{Ag}$, and $\mathrm{CV}$ molecules ZnONRs@Ag/CV system; and iii) the chemical interactions semiconductor and molecules associated with CM. Therefore, heterostructure material-based sensing platforms may pave a facile route for highly sensitive detection at trace levels in practical application. 


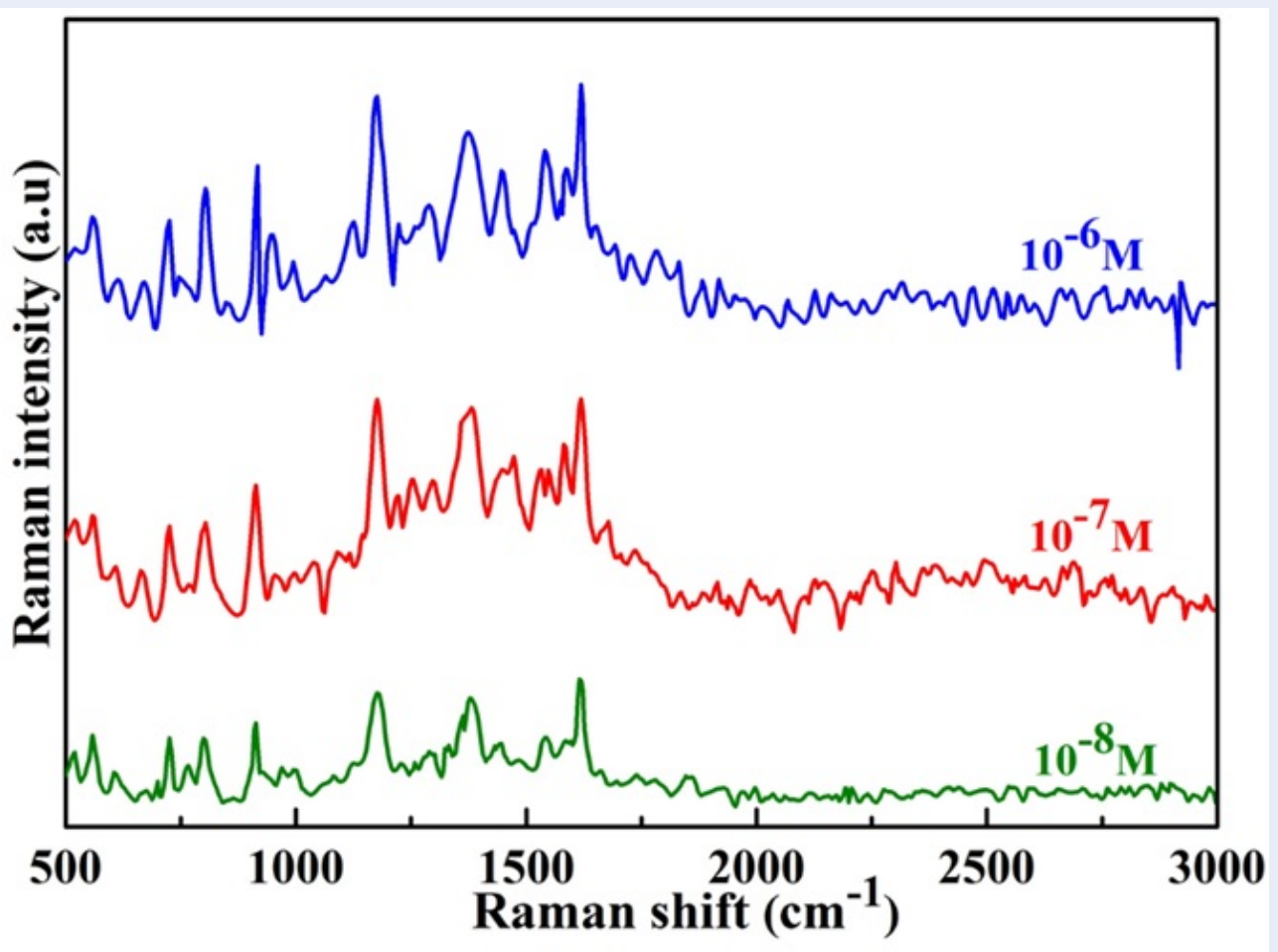

Figure 6: Raman spectra of CV molecules obtained from the ZnONRs@Ag-1.0 platform at different concentrations from $10^{-6}$ to $10^{-8} \mathrm{M}$.

\section{ABBREVIATIONS}

CM: chemical mechanism

EM: electromagnetic mechanism

ZnO: Zinc oxide

\section{COMPETING INTERESTS}

The authors declare that there is no conflict of interest regarding the publication of this article.

\section{AUTHORS' CONTRIBUTIONS}

Ton Nu Quynh Trang has conceived of the present idea, carried out and written the manuscript with support from Vu Thi Hanh Thu

Le To Cam Huong and Thai Duong have done all experiments.

\section{ACKNOWLEDGMENTS}

This research is funded by Vietnam National University Ho Chi Minh City (VNU-HCM) under grant number VL2019-18-01.

\section{REFERENCES}

1. Pérez-Jiménez Al, Lyu D, Lu Z, Liu G, Ren B. Surface-enhanced Raman spectroscopy: benefits, trade-offs and future developments. Chemical Science. 2020;11(18):4563-77;Available from: https://doi.org/10.1039/D0SC00809E
2. Moskovits M. Surface roughness and the enhanced intensity of Raman scattering by molecules adsorbed on metals. The Journal of Chemical Physics. 1978;69(9):4159-61;Available from: https://doi.org/10.1063/1.437095.

3. Korkmaz A, Kenton M, Aksin G, Kahraman M, WachsmannHogiu S. Inexpensive and flexible SERS substrates on adhesive tape based on biosilica plasmonic nanocomposites. ACS Applied Nano Materials. 2018;1(9):5316-26;Available from: https: //doi.org/10.1021/acsanm.8b01336.

4. Pérez-Jiménez Al, Lyu D, Lu Z, Liu G, Ren B. Surface-enhanced Raman spectroscopy: benefits, trade-offs and future developments. Chemical Science. 2020;11(18):4563-77;Available from: https://doi.org/10.1039/D0SC00809E.

5. Langer J, Jimenez de $A D$, Aizpurua J, Alvarez-Puebla RA, Auguié B, Baumberg JJ, Bazan GC, Bell SE, Boisen A, Brolo AG, Choo J. Present and future of surface-enhanced Raman scattering. ACS nano. 2019;14(1):28-117;PMID: 31478375. Available from: https://doi.org/10.1021/acsnano.9b04224.

6. Lindquist NC, de Albuquerque CD, Sobral-Filho RG, Paci I, Brolo AG. High-speed imaging of surface-enhanced Raman scattering fluctuations from individual nanoparticles. Nature nanotechnology. 2019;14(10):981-7;PMID: 31527841. Available from: https://doi.org/10.1038/s41565-019-0535-6.

7. Trang TN, Vinh LQ, Doanh TT, Thu VT. Structure-adjustable colloidal silver nanoparticles on polymers grafted cellulose paper-based highly sensitive and selective SERS sensing platform with analyte enrichment function. Journal of Alloys and Compounds. 2021:159158;Available from: https://doi.org/10. 1016/j.jallcom.2021.159158.

8. Chaudhari K, Ahuja T, Murugesan V, Subramanian V, Ganayee MA, Thundat T, Pradeep T. Appearance of SERS activity in single silver nanoparticles by laser-induced reshaping. Nanoscale. 2019;11(1):321-30;PMID: 30534777. Available from: https://doi.org/10.1039/C8NR06497K. 


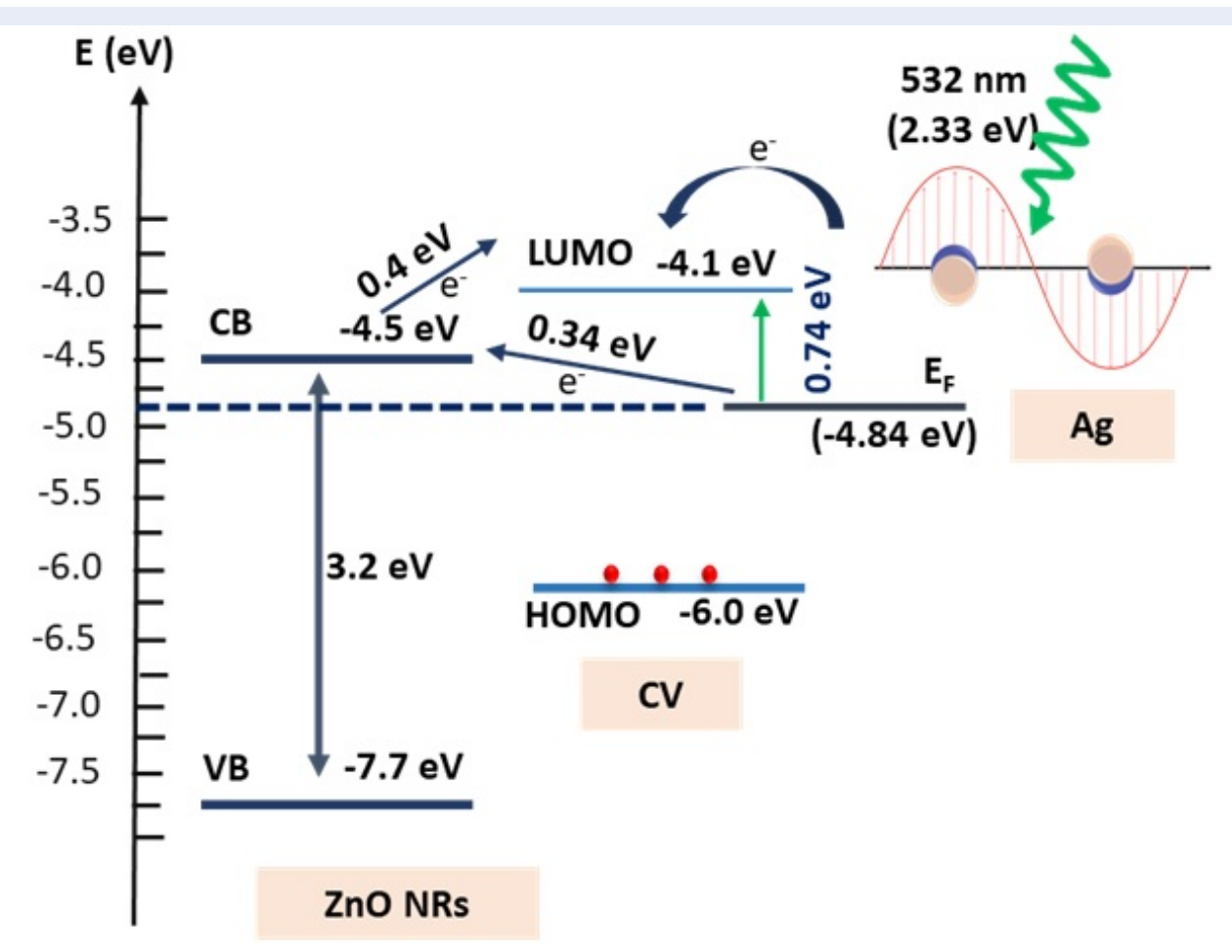

Figure 7: Energy band structure illustrating of charge transfer transition of the ZnONRs/Agand CV molecules.

9. Guo $\mathrm{H}$, Zhao $\mathrm{A}$, $\mathrm{He} \mathrm{O}$ Chen $\mathrm{P}$, Wei $\mathrm{Y}$, Chen $\mathrm{X}, \mathrm{Hu} \mathrm{H}$ Wang M, Huang H, Wang R. Multifunctional Fe3O4@mTiO2@ noble metal composite NPs as ultrasensitive SERS substrates for trace detection. Arabian Journal of Chemistry. 2019;12(8):2017-27;Available from: https://doi.org/10.1016/j. arabjc.2019.01.007.

10. Li X, Zhu J, Wei B. Hybrid nanostructures of metal/twodimensional nanomaterials for plasmon-enhanced applications. Chemical Society Reviews. 2016;45(11):314587;PMID: 27048993. Available from: https://doi.org/10.1039/ C6CS00195E.

11. Chen HY, Lin MH, Wang CY, Chang YM, Gwo S. Large-scale hot spot engineering for quantitative SERS at the singlemolecule scale. Journal of the American Chemical Society. 2015;137(42):13698-705;PMID: 26469218. Available from: https://doi.org/10.1021/jacs.5b09111.

12. Tieu DT, Trang TN, Thu VT. Assembly engineering of Ag@ ZnO hierarchical nanorod arrays as a pathway for highly reproducible surface-enhanced Raman spectroscopy applications. Journal of Alloys and Compounds. 2019;808:151735;Available from: https://doi.org/10.1016/j.jallcom.2019.151735.

13. Kim W, Lee SH, Kim SH, Lee JC, Moon SW, Yu JS, Choi S. Highly reproducible Au-decorated $\mathrm{ZnO}$ nanorod array on a graphite sensor for classification of human aqueous humors. ACS applied materials \& interfaces. 2017;9(7):5891-9;PMID: 28156092. Available from: https://doi.org/10.1021/acsami. $6 \mathrm{~b} 16130$.

14. Trang TN, Phan TB, Nam ND, Thu VT. In situ charge transfer at the Ag@ ZnO photoelectrochemical interface toward the high photocatalytic performance of $\mathrm{H} 2$ evolution and $\mathrm{RhB}$ degradation. ACS applied materials \& interfaces. 2020;12(10):12195206;PMID: 32013392. Available from: https://doi.org/10.1021/ acsami.9b15578.
15. Jiang $X$, Sun $X$, Yin D, Li X, Yang $M$, Han X, Yang L, Zhao B. Recyclable Au-TiO 2 nanocomposite SERS-active substrates contributed by synergistic charge-transfer effect. Physical Chemistry Chemical Physics. 2017;19(18):11212-9;PMID: 28405659. Available from: https://doi.org/10.1039/C7CP01610G.

16. Zhu Q, Xu C, Wang D, Liu B, Qin F, Zhu Z, Liu Y, Zhao X, Shi Z. Femtomolar response of a plasmoncoupled $\mathrm{ZnO} / g$ raphene/silver hybrid whispering-gallery mode microcavity for SERS sensing. Journal of Materials Chemistry C. 2019;7(9):2710-6;Available from: https://doi.org/10.1039/C8TC06305B.

17. Dong J, Huang J, Wang A, Biesold-McGee GV, Zhang X, Gao S, Wang S, Lai Y, Lin Z. Vertically-aligned Ptdecorated MoS2 nanosheets coated on $\mathrm{TiO} 2$ nanotube arrays enable high-efficiency solar-light energy utilization for photocatalysis and self-cleaning SERS devices. Nano Energy. 2020;71:104579;Available from: https://doi.org/10.1016/j.nanoen.2020.104579.

18. Chen H, Das A, Bi L, Choi N, Moon JI, Wu Y, Park S, Choo J. Recent advances in surface-enhanced Raman scattering-based microdevices for point-of-care diagnosis of viruses and bacteria. Nanoscale. 2020;12(42):21560-70;PMID: 33094771. Available from: https://doi.org/10.1039/D0NR06340A.

19. Zhou J, et al. Plasmon-induced hot electron transfer in AuZnO heterogeneous nanorods for enhanced SERS. Nanoscale. 2019;11(24):11782-11788. PMID: 31184351. Available from: https://doi.org/10.1039/C9NR02969A.

20. Korepanov VI, Chan SY, Hsu HC, Hamaguchi HO. Phonon confinement and size effect in Raman spectra of ZnO nanoparticles. Heliyon. 2019;5(2):e01222;PMID: 30828658. Available from: https://doi.org/10.1016/j.heliyon.2019.e01222.

21. Shi G, et al. A novel natural SERS system for crystal violet detection based on graphene oxide wrapped Ag micro-islands 
substrate fabricated from Lotus leaf as a template. Applied Surface Science. 2018;459:802-811. Available from: https: //doi.org/10.1016/j.apsusc.2018.08.065.

22. Harraz FA, Ismail AA, Bouzid H, Al-Sayari SA, Al-Hajry A, Al-Assiri MS. Surface-enhanced Raman scattering (SERS)- active substrates from silver plated-porous silicon for detection of crystal violet. Applied Surface Science. 2015;331:2417;Available from: https://doi.org/10.1016/j.apsusc.2015.01.042. 